\title{
Profile of Polatuzumab Vedotin in the Treatment of Patients with Relapsed/Refractory Non-Hodgkin Lymphoma: A Brief Report on the Emerging Clinical Data
}

Yazeed Sawalha (D) Kami Maddocks (D)

Department of Internal Medicine, Division of Hematology, Arthur G. James Comprehensive Cancer Center, The Ohio State University Wexner Medical Center, Columbus, OH, USA
Correspondence: Yazeed Sawalha Department of Internal Medicine, Division of Hematology, The Ohio State University Comprehensive Cancer Center - Arthur G. James Cancer Hospital, A350B Starling-Loving Hall, 320 W. I0th Ave., Columbus, $\mathrm{OH} 43210$, USA $\mathrm{Tel}+\mid$ 614-293-0837

Fax + 614-293-7526

Email Yazeed.sawalha@osumc.edu
This article was published in the following Dove Press journal: OncoTargets and Therapy

\begin{abstract}
Polatuzumab vedotin is an anti-CD79b antibody conjugated to monomethyl auristatin $\mathrm{E}$ that has shown significant clinical activity in follicular and diffuse large B-cell lymphoma (DLBCL) and is currently FDA-approved in combination with bendamustine and rituximab for patients with relapsed/refractory DLBCL. This review article summarizes data from clinical trials of polatuzumab and discusses its current role and future directions in the treatment of patients with B-cell non-Hodgkin lymphoma.

Methods: We conducted a literature search in PubMed and Google Scholar from inception to January 2020, using the following terms: polatuzumab and CD79. We also reviewed the package insert and available abstracts and posters presented at national and international meetings.
\end{abstract}

Keywords: CD79, antibody-drug conjugate, polatuzumab, clinical trials, novel

\section{Introduction}

Antibody-drug conjugates (ADCs) are monoclonal antibodies conjugated to cytotoxic payloads and directed toward tumor-associated antigens. Such a strategy combines the high selectivity and favorable pharmacokinetic profile of monoclonal antibodies with the high antitumor potency of the cytotoxic payload.

Polatuzumab vedotin (POLIVYTM, Genentech) (pola) is an ADC consisting of a humanized anti-CD79b monoclonal antibody covalently attached via a proteasecleavable linker to monomethyl auristatin E (MMAE), a microtubule-disrupting antimitotic agent (Figure 1). ${ }^{1}$ Pola carries an average of 3.5 molecules of MMAE for each anti-CD79b antibody molecule. Once internalized, the linker is cleaved and MMAE is released resulting in microtubule network disruption and inhibition of cell division and growth. In June 2019, the US Food and Drug Administration (FDA) approved pola in combination with bendamustine and rituximab (BR) for patients with relapsed/refractory diffuse large B-cell lymphoma (DLBCL) after at least two prior therapies. Pola has also shown significant clinical activity in patients with follicular lymphoma (FL). Several other ADCs have proven to be effective in hematologic malignancies, with brentuximab vedotin (anti-CD30), inotuzumab ozogamicin (anti-CD22), and moxetumomab pasudotox (anti-CD22) currently approved by the FDA for use in lymphoid malignancies, while many others are being developed or tested in clinical trials. 


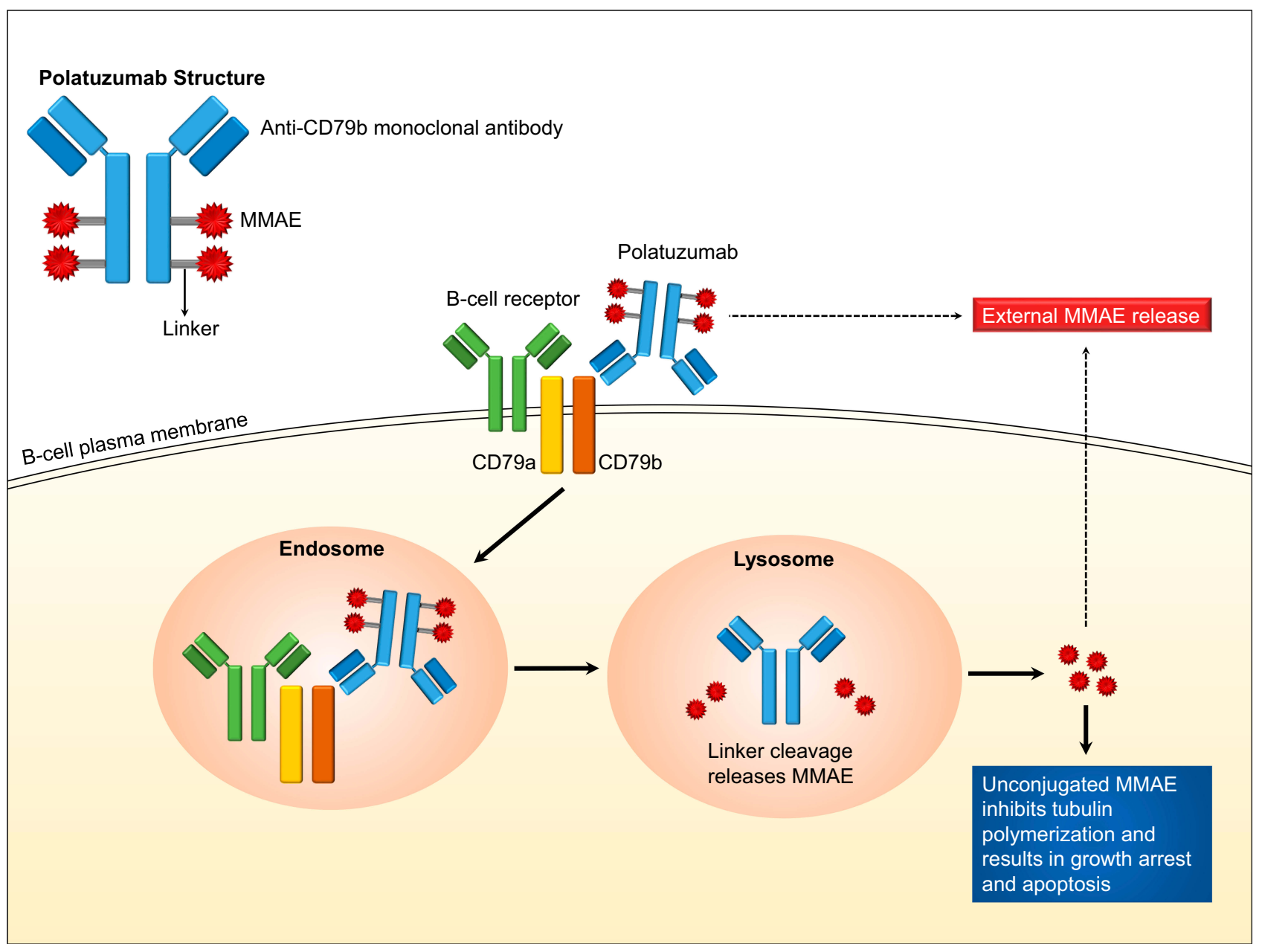

Figure I Polatuzumab structure and mechanism of action. Polatuzumab is composed of an anti-CD79b monoclonal antibody conjugated to monomethyl auristatin $\mathrm{E}$ (MMAE) via a protease-cleavable linker. After binding to CD79b, the complex is internalized by endocytosis and directed to lysosomes where the linker is cleaved and MMAE released to the cytosol. Free MMAE inhibits microtubule formation and results in cell growth arrest and apoptosis. Extracellular MMAE release can occur leading to systemic adverse events.

\section{The Target - CD79b}

The B-cell receptor (BCR) is composed of a membrane immunoglobulin (IgM) noncovalently attached to CD79. ${ }^{2}$ During B-cell development, CD79 expression precedes immunoglobulin heavy-chain gene rearrangement and CD20 expression and disappears in the plasma-cell stage of B-cell differentiation. ${ }^{3}$ CD79 functions as the signaling component of the BCR and is composed of a heterodimer of CD79a and CD79b, with each subunit containing a single extracellular immunoglobulin domain, a transmembrane domain, and an intracellular signaling domain. $^{2}$ Antigen binding to the BCR initiates signaling events that include internalization of the ligand-receptor complexes and, as part of MHC class II antigen presentation by B cells, trafficking these complexes to lysosomes. Both CD79a and CD79b can induce internalization of the $\mathrm{BCR}$ but $\mathrm{CD} 79 \mathrm{~b}$ has the dominant role. ${ }^{4,5}$ In this manner, pola bound to $\mathrm{CD} 79 \mathrm{~b}$ is internalized and directed to lysosomal compartments, which contain proteases that can cleave the linker and release the cytotoxic payload (Figure 1). ${ }^{4} \mathrm{CD} 79 \mathrm{~b}$ is an ideal target for ADC-based therapy given its high expression on most types of B-cell non-Hodgkin lymphoma (NHL) and chronic lymphocytic leukemia (CLL) and limited expression on normal cells (restricted to pre-B and mature B cells). ${ }^{1,6-8}$ Notably, recovery of circulating $B$ cells was observed following administration of pola in non-human primates as CD79b is not expressed on hematopoietic stem cells. ${ }^{9} C D 79 b$ mutations, which occur in up to $25 \%$ of the activated B-cell (ABC) subtype of DLBCL, ${ }^{10}$ did not affect the internalization rates of pola compared with wild-type CD79b in preclinical studies. ${ }^{8}$ Targeting CD79 with unconjugated antibodies resulted in minimal antibodydependent cell-mediated or complement-dependent 
cytotoxicity, ${ }^{4,8,9}$ suggesting that the main therapeutic effect of anti-CD79b ADCs is mediated by MMAE.

Based on CD79b cell-surface expression by flow cytometry, Dornan et al showed that a minimal threshold of CD79b expression was required for the in vitro activity of anti-CD79b ADC in NHL cell lines, and that the lack of CD79b expression was the primary mechanism of resistance. ${ }^{1}$ Applying this CD79b cell-surface expression threshold on 292 patient samples, $90 \%$ or more of DLBCL, FL, marginal zone lymphoma, hairy cell leukemia, and mantle cell lymphoma (MCL) cases expressed sufficient $\mathrm{CD} 79 \mathrm{~b}$ to be responsive to the $\mathrm{ADC}$, compared with only $23 \%$ of CLL cases. ${ }^{1}$ Other studies showed that CD79b expression in CLL was weaker than in other closely related lymphoid malignancies such as Richter's transformation (CLL transformation to DLBCL) and B-cell prolymphocytic leukemia. ${ }^{6,7,11}$ In the same study, Dornan et al also demonstrated that sufficient CD79b expression persisted in the majority of FL (87\%) and DLBCL (77\%) cases that relapsed after treatment with chemotherapy. ${ }^{1}$ Furthermore, the majority of DLBCL cases $(92 \%, n=24)$ expressed CD79b by immunohistochemistry (IHC) with no significant difference in expression among the three molecular subtypes of DLBCL (germinal center B-cell (GCB), ABC, unclassifiable) based on cell-of-origin (COO) as determined by gene expression profiling (GEP). ${ }^{1}$ Overall, these findings suggested that most NHL types have sufficient $\mathrm{CD} 79 \mathrm{~b}$ expression to be susceptible for anti-CD79b ADCs.

\section{Clinical Trials of Polatuzumab Monotherapy or in Combination with Rituximab}

The first-in-human Phase 1 clinical trial of pola comprised two dose-escalation cohorts in patients with relapsed/refractory NHL $(n=34)$ or CLL $(n=18)$ followed by two doseexpansion cohorts in NHL; one with pola alone $(n=34)$ and one in combination with rituximab (R-pola, $n=9)($ Table 1$) .^{12}$ An expansion cohort in CLL was not pursued due to the lack of activity in the dose-escalation cohort. Overall, the trial included 40 patients with DLBCL, 30 with indolent NHL, 18 with CLL, and 7 with MCL. For patients with DLBCL or indolent NHL, the median age was 67 years (range, 20-81 in DLBCL and 41-86 in indolent NHL), and the majority received $\geq 3$ prior lines of therapy ( $88 \%$ of DLBCL and $70 \%$ of indolent NHL) and were refractory to last therapy (78\% of DLBCL and $53 \%$ of indolent NHL). Patients received pola at escalating doses of 0.1 to $2.4 \mathrm{mg} / \mathrm{kg}$ every 3 weeks until disease progression, unacceptable toxicity, or patient/physician's decision. Only one dose-limiting toxicity was reported during dose escalation (grade 4 neutropenia at the $2.4 \mathrm{mg} / \mathrm{kg}$ dose level). Twenty-seven patients with DLBCL, 16 with indolent NHL, and 2 with MCL received the recommended Phase 2 dose (RP2D) of $2.4 \mathrm{mg} / \mathrm{kg}$ for a median of 6 cycles (range, 1-17). The most common grade 3-4 adverse events (AEs) were neutropenia (40\%), anemia (11\%), and peripheral neuropathy (PN) (9\%). In the 9 patients treated with R-pola, grade 3-4 neutropenia and neutropenic fever occurred in $56 \%$ and $22 \%$, respectively. Of all patients treated on trial, three patients died from treatment-related infections including lung infection, Serratia spp pneumonia and Clostridium difficile sepsis. Approximately half of the patients (51\%) discontinued treatment due to AEs including PN and paresthesia in $29 \%$. No other AE led to treatment discontinuation in more than one patient. $\mathrm{PN}$ occurred in $51 \%$ of the patients treated at the RP2D and was grade $1-2$ in $42 \%$ and grade $3-4$ in $9 \%$. The PN was predominantly sensory except for 2 cases of motor neuropathy, had a median time to onset of 3.5 months, and resolved in $54 \%$ of the cases that required dose reductions or treatment delays/discontinuations. Notably, $17 \%$ of patients had grade 1 baseline PN prior to starting pola. In the evaluable patients treated at the RP2D, the objective response rate (ORR) was $56 \%$ in DLBCL (14/25) including complete response (CR) in $16 \%$ and $47 \%$ in indolent NHL (7/15) including CR in $20 \%$. The two patients with MCL treated at the RP2D had a partial response. The median progression-free survival (PFS) and duration of response (DOR) were both 5 months in DLBCL, and 8 months and not reached in indolent NHL, respectively. In the 9 patients treated with R-pola ( 5 indolent NHL, 3 MCL, and 1 transformed FL), 7 patients (78\%) had an objective response including 2 with $\mathrm{CR}(22 \%)$. The median PFS and DOR were 13 and 12 months, respectively. In CLL, the maximum tolerated dose was $1 \mathrm{mg} / \mathrm{kg}$ (two of five patients treated at $1.8 \mathrm{mg} / \mathrm{kg}$ had dose-limiting toxicities of grade 4 neutropenia and grade 4 fungal infection). None of the 18 patients with CLL had an objective response. Pola's lack of efficacy in CLL was attributed to faster clearance and lower exposure of pola in CLL compared with NHL. There were no data on $\mathrm{CD} 79 \mathrm{~b}$ expression levels in patients with CLL or NHL, and CD79b expression was not a prerequisite for study entry. ${ }^{12}$

The ROMULUS phase 2 clinical trial randomized patients with relapsed/refractory FL or DLBCL to rituximab plus either pola (R-pola) or pinatuzumab vedotin (Table 1). ${ }^{13}$ Pinatuzumab vedotin is a similar ADC that is composed of an anti-CD22 monoclonal antibody 
Table I Results of Phase I/2 Clinical Trials of Polatuzumab

\begin{tabular}{|c|c|c|c|c|c|}
\hline $\begin{array}{l}\text { Study Reference } \\
\text { (Phase) }\end{array}$ & $\begin{array}{l}\text { NHL Subtype } \\
\text { (n) }\end{array}$ & Treatment & $\begin{array}{l}\text { ORR }^{\mathrm{a}}(\mathrm{CR}) \\
\text { at RP2D }\end{array}$ & $\begin{array}{l}\text { PFS/DOR/OS } \\
\text { Median (Months) }\end{array}$ & $\begin{array}{l}\text { Grade 3-4 AEs of Interest/Significance } \\
\text { (at RP2D) }\end{array}$ \\
\hline \multirow[t]{5}{*}{ NCTOI $290549^{12}$ (I) } & \multirow{5}{*}{$\begin{array}{l}\text { R/R: } \\
\text { DLBCL (40) } \\
\text { iNHL (30) } \\
\text { CLL (18) } \\
\text { MCL (7) }\end{array}$} & \multirow[t]{4}{*}{ Pola } & $56 \%(16 \%)$ & $5 / 5 /$ not reported & \multirow{5}{*}{$\begin{array}{l}\text { - Neutropenia } 40 \% \text {, anemia } 1 \mathrm{I} \% \\
\text { - } 3 \text { patients died from treatment-related } \\
\text { infections } \\
\text { - } 51 \% \text { discontinued treatment due to AEs } \\
\text { - } \mathrm{PN} \text { in } 51 \% \text { : GI-2 } 42 \% \text {, G3-4 } 9 \% \text {; resolved } \\
\text { in } 54 \%\end{array}$} \\
\hline & & & DLBCL $(n=25)$ & & \\
\hline & & & $47 \%(20 \%)$ & $8 /$ not reached/not & \\
\hline & & & iNHL $(n=15)$ & reported & \\
\hline & & R-pola & $\begin{array}{l}78 \%(22 \%) \\
(n=9)\end{array}$ & $13 / 12 /$ not reported & \\
\hline \multirow[t]{2}{*}{$\begin{array}{l}\text { ROMULUS }{ }^{13} \\
\text { (2) }\end{array}$} & R/R DLBCL (39) & R-pola & $54 \%(21 \%)$ & $6 / 13 / 20$ & $\begin{array}{l}\text { - Neutropenia } 23 \% \text {, anemia } 8 \% \text {, diarrhea } \\
8 \% \\
\text { - } 26 \% \text { discontinued treatment due to AEs } \\
\text { - PN in } 56 \% \text { : G2 } 26 \% \text {, G3 } 10 \%\end{array}$ \\
\hline & R/R FL (20) & R-pola & $70 \%(45 \%)$ & I5/9/Not reached & $\begin{array}{l}\text { - Neutropenia } 15 \% \text {, diarrhea } 10 \% \\
\text { - } 55 \% \text { discontinued treatment due to AEs } \\
\text { - PN in } 95 \% \text {; G2 } 75 \% \text {, G3 } 5 \%\end{array}$ \\
\hline \multirow{5}{*}{$\begin{array}{l}\mathrm{GO} 29365^{14,15} \\
(\mathrm{Ib} / 2)\end{array}$} & R/R DLBCL (27) & Pola-BG & $41 \%(30 \%)$ & $6 / 28 / 11$ & \multirow{5}{*}{$\begin{array}{l}\text { Pola-BR in DLBCL: } \\
\text { - Anemia } 28 \% \text {, thrombocytopenia } 41 \% \text {, } \\
\text { neutropenia } 46 \% \text {, NF } 10 \% \\
\text { - } 33 \% \text { discontinued treatment due to AEs } \\
\text { - } \mathrm{PN} \text { in } 44 \% \text {; GI } 28 \% \text {, G2 } 15 \% \text {, resolved in } \\
59 \%\end{array}$} \\
\hline & \multirow{2}{*}{$\begin{array}{l}\mathrm{R} / \mathrm{R} \text { DLBCL }(80) \\
\text { randomized }\end{array}$} & Pola-BR & $45 \%(40 \%)$ & $10 / 13 / 12$ & \\
\hline & & $B R$ & $18 \%(18 \%)$ & $4 / 8 / 5$ & \\
\hline & \multirow[t]{2}{*}{$\begin{array}{l}\mathrm{R} / \mathrm{R} \mathrm{FL}(80) \\
\text { randomized }\end{array}$} & Pola-BR & $77 \%$ (69\%) & $\begin{array}{l}\text { I7/not reported/not } \\
\text { reached }\end{array}$ & \\
\hline & & BR & $73 \%(63 \%)$ & $\begin{array}{l}17 / \text { not reported/not } \\
\text { reached }\end{array}$ & \\
\hline $\begin{array}{l}\text { NCT03274492 } 22 \\
(\mathrm{Ib} / 2)\end{array}$ & $\begin{array}{l}\text { Untreated } \\
\text { DLBCL (75) }\end{array}$ & $\begin{array}{l}\text { Pola }+ \\
\text { R-CHPI } \\
\text { G-CHP }\end{array}$ & $89 \%(77 \%)$ & $\begin{array}{l}\text { 24-month PFS of } \\
83 \%\end{array}$ & $\begin{array}{l}\text { - Neutropenia } 30 \% \text {, neutropenic fever } 8 \% \text {, } \\
\text { thrombocytopenia } 9 \% \\
\text { - PN in } 41 \% \text {; GI } 27 \% \text {, G2 II\%, G3 3\%; } \\
\text { resolved in } 74 \%\end{array}$ \\
\hline $\begin{array}{l}\text { GO29834 } \\
(\mathrm{Ib} / 2)\end{array}$ & $\mathrm{R} / \mathrm{R} \mathrm{FL}$ & Pola-G-len & $76 \%(63 \%)$ & $\begin{array}{l}\text { 12-month PFS of } \\
83 \%\end{array}$ & $\begin{array}{l}\text { - Neutropenia } 50 \% \text {, thrombocytopenia } \\
23 \% \text {, infections } 16 \% \text {, anemia } 14 \% \text {. } \\
\text { - } 30 \% \text { discontinued treated due to AEs } \\
\text { - } \mathrm{PN} \text { in } 30 \%\end{array}$ \\
\hline
\end{tabular}

Notes: ${ }^{a}$ Responses were assessed by positron emission tomography-computed tomography (PET-CT) in ROMULUS, G029365, NCT03274492, and GO29834; and CT or PET-CT in NCTOI290549.

Abbreviations: AEs, adverse events; BR, bendamustine + rituximab; CLL, chronic lymphocytic leukemia; CR, complete response; DLBCL, diffuse large B-cell lymphoma; DOR, duration of response; FL, follicular lymphoma; G, grade; G-CHP, obinutuzumab, cyclophosphamide, doxorubicin, prednisone; iNHL, indolent non-Hodgkin lymphoma; MCL, mantle cell lymphoma; ORR, objective response rate; OS, overall survival; PFS, progression-free survival; PN, peripheral neuropathy; Pola, polatuzumab; Pola-G-Len, polatuzumab, obinutuzumab, lenalidomide; R-CHP, rituximab, cyclophosphamide, doxorubicin, prednisone; RP2D, recommended phase 2 dose.

conjugated to MMAE. Both pola and pinatuzumab were developed by Genentech, but pola was selected for further development based on the longer duration of response achieved with R-pola in this study. Patients received rituximab plus one of the ADCs at a dose of $2.4 \mathrm{mg} / \mathrm{kg}$ every 3 weeks until disease progression or unacceptable toxicity for up to 1 year. For the 39 patients with DLBCL randomized to R-pola, median age was 68 years (range, 55-77), Eastern Cooperative Oncology Group performance status
(ECOG PS) was $0-1$ in $95 \%$ and 2 in 5\%, median number of prior treatments was 3 (range, 2-4), 18\% underwent prior autologous stem cell transplantation (SCT), and $80 \%$ were refractory to last treatment. In this cohort of patients with DLBCL, R-pola resulted in 54\% ORR including 21\% $\mathrm{CR}$ and median PFS, DOR, and overall survival (OS) of 6 , 13 , and 20 months, respectively. For the 20 patients with FL randomized to R-pola, median age was 67 years (range, 59-74), ECOG PS was $0-1$ in $95 \%$ and 2 in $5 \%$, 
no patients had bulky disease, median number of prior treatments was 2 (range, 2-4), 25\% were refractory to rituximab, and $35 \%$ were refractory to last treatment. In this cohort of patients with FL, R-pola resulted in $70 \%$ ORR including $45 \% \mathrm{CR}$ and median PFS and DOR of 15 and 9 months, respectively, whereas the median OS was not reached. None of the 6 patients ( 5 DLBCL, 1 FL) who crossed over to treatment with the other ADC following disease progression (including 4 patients crossing over from R-pinatuzumab to R-pola) responded, suggesting that the main mechanism of resistance was to MMAE rather than to the antibody target. The most common grade 3-4 AEs with R-pola in DLBCL were neutropenia $(23 \%)$, anemia $(8 \%)$, and diarrhea $(8 \%)$, and in FL were neutropenia $(15 \%)$ and diarrhea $(10 \%)$. PN occurred in $56 \%$ of the patients with DLBCL (grade 2 in $26 \%$ and grade 3 in 10\%) and $95 \%$ of the patients with FL (grade 2 in $75 \%$ and grade 3 in 5\%). PN developed in a median of 2-3 months, lasted for a median of 14-17 months, and led to treatment discontinuation in $18 \%$ and $55 \%$ of the patients with DLBCL and FL, respectively. Notably, grade $1 \mathrm{PN}$ was present at study entry in $39 \%$ and $25 \%$ of the patients with DLBCL and FL, respectively. The higher incidence of $\mathrm{PN}$ in FL compared with DLBCL was likely related to the longer duration of treatment with R-pola in FL (median of 10.5 vs 6 cycles).

Taken together, these two clinical trials showed that pola monotherapy or in combination with rituximab had encouraging activity in patients with heavily pretreated DLBCL and FL, but needed to be combined with other active agents to improve the depth and duration of response. Because PN occurred in more than half of the patients treated with pola $2.4 \mathrm{mg} / \mathrm{kg}$ and frequently led to treatment discontinuation, the maximum dose of pola in the dose-escalation portion of subsequent combination trials was set at $1.8 \mathrm{mg} / \mathrm{kg}$.

\section{In Combination with Chemotherapy or Targeted Agents \\ Relapsed/Refractory Setting}

The GO29365 phase 1b/2 clinical trial combined pola with bendamustine plus either rituximab (pola-BR) or obinutuzumab (pola-BG) in patients with relapsed/refractory DLBCL (Table 1). ${ }^{14}$ Six patients were treated with pola$\mathrm{BR}$ and 6 with pola-BG in the safety run-in phase followed by an expansion cohort of 21 patients treated with pola$\mathrm{BG}$ and a cohort of 80 patients randomized to treatment with BR alone or pola-BR. Notable inclusion criteria were ECOG PS 0-2, receipt of at least 1 prior therapy, baseline $\mathrm{PN} \leq$ grade 1, and ineligibility for SCT (prior autologous SCT was allowed). Patients with transformed FL were excluded. Treatment consisted of six 21-day cycles of bendamustine $\left(90 \mathrm{mg} / \mathrm{m}^{2}\right)$ on days $2+3$ of cycle 1 and days $1+2$ of subsequent cycles; rituximab on day 1 or obinutuzumab on days 1,8 , and 15 of cycle 1 and day 1 of subsequent cycles; and pola $1.8 \mathrm{mg} / \mathrm{kg}$ on day 2 of cycle 1 and day 1 of subsequent cycles. Focusing on the 80 patients randomized to $\mathrm{BR}$ or pola-BR, median age was 71 years (range, 30-84) for the BR cohort and 67 years (range, 33-86) for the pola-BR cohort. Most patients had ECOG PS of $0-1$ (83\% pola-BR and $78 \%$ BR). The median number of prior treatments was 2, with most patients being refractory to last therapy $(85 \% \mathrm{BR}, 75 \%$ pola-BR). Patients were ineligible for autologous SCT most commonly due to age ( $48 \% \mathrm{BR}, 33 \%$ pola-BR) and insufficient response to salvage therapy (30\% pola-BR and $23 \% \mathrm{BR}) ; 15 \%$ of the BR cohort and $25 \%$ of the pola-BR cohort experienced treatment failure with prior autologous SCT. Patients with double- and triple-hit lymphomas were allowed but none were included. Compared with BR, pola$\mathrm{BR}$ resulted in higher end-of-treatment (EOT) ORR (45\% vs $18 \%$ ) and CR rate (the primary endpoint: $40 \%$ vs $18 \%$ ) as well as higher best response (63\% vs $25 \%)$ and best CR rates ( $50 \%$ vs $23 \%$ ), as reviewed by an independent review committee (IRC). Although the clinical trial was not powered to detect a survival benefit, the pola-BR arm had significantly better IRC-assessed PFS (median of 10 vs 4 months; hazard ration (HR) $0.36,95 \%$ confidence interval (CI) $0.21-0.63$ ) and OS (median of 12 vs 5 months; HR 0.42 , 95\% CI 0.24-0.75). The median DOR was longer with pola-BR (13 vs 8 months) but the difference was not statistically significant. Seven patients treated with polaBR (18\%) had ongoing responses for more than 20 months, including one patient who underwent allogeneic SCT. Bearing in mind the relatively small sample size, pola-BR demonstrated an OS benefit regardless of age ( $<65$ vs $\geq 65$ years), refractoriness to last therapy, number of prior lines of therapy ( 1 vs $\geq 2$ ), prior autologous SCT, or DLBCL subtype based on COO by GEP (GCB vs ABC) or $\mathrm{MYC/BCL2}$ double-expression status (available for $\mathrm{n}=62$, present in $\mathrm{n}=17$ ). Only $46 \%$ of patients in the polaBR cohort and $23 \%$ in the BR cohort completed 6 cycles of treatment, with the primary reason for treatment discontinuation being AEs in the pola-BR cohort (33\% vs $10 \%$ with $\mathrm{BR}$ ), and disease progression in the BR cohort 
(54\% vs $15 \%$ with pola-BR). Grade 3-4 anemia, thrombocytopenia, and neutropenia were more common with pola-BR $(28 \%, 41 \%$, and $46 \%$ vs $18 \%, 23 \%$, and $33 \%$, respectively), but the rate of neutropenic fever was similar in the two cohorts ( $10 \%$ pola-BR, $13 \%$ BR). Most patients received at least one dose of granulocyte colonystimulating factor (G-CSF) (72\% pola-BR, 62\% BR). Four patients in each arm died of infection. Diarrhea of all grades was more common with pola-BR (39\% vs $28 \%$, grade $3-4$ of $3 \%$ in each). PN occurred in $44 \%$ of patients treated with pola-BR (28\% grade 1, $15 \%$ grade 2$)$ and resolved in $59 \%$ of the cases. The baseline characteristics of the 27 patients treated with pola-BG were similar to those treated with pola-BR. Pola-BG was not superior to pola-BR, with EOT ORR of $41 \%$ include CR rate of $30 \%$, best ORR of $48 \%$, and best CR rate of $37 \%$, while the median PFS and OS were 6 and 11 months, respectively. ${ }^{14}$ Based on these results, in June 2019, the FDA granted accelerated approval to pola $(1.8 \mathrm{mg} / \mathrm{kg})$ in combination with BR every 3 weeks for 6 cycles in patients with relapsed/refractory DLBCL after at least two prior therapies.

In addition to DLBCL, the GO29365 clinical trial included a randomly assigned cohort of 80 patients with relapsed/refractory FL treated with pola-BR or BR alone, with interim data presented at the American Society of Clinical Oncology 2018 Annual Meeting (data cut-off October 2017) (Table 1). ${ }^{15}$ Pola-BR and BR were given at the same dose and schedule as in the DLBCL cohort but treatment cycles were 28 days in duration. The median age was 63 years (range, 39-80) for the BR cohort and 65 years (range, 43-74) for the pola-BR cohort. Most patients had ECOG PS of $0-1$ ( $88 \%$ BR, 92\% pola-BR) and nonbulky disease ( $88 \%$ BR, $85 \%$ pola-BR). The median number of prior therapies was 2 (range, 1-5) for each cohort, with disease refractory to last treatment in $42 \%$ of the BR cohort and $41 \%$ of the pola-BR cohort, and disease progression within 24 months of initial diagnosis (POD24) in $24 \%$ of the BR cohort and $31 \%$ the pola-BR cohort. The EOT ORR and CR rates were similar between the two cohorts $(77 \%$ and $69 \%$ with pola-BR and $73 \%$ and $63 \%$ with BR, respectively). With the limited follow-up duration of 15 months, the median PFS and OS were also similar (17 months and not reached for each cohort, respectively). ${ }^{15}$

Several clinical trials have established lenalidomide plus rituximab as an effective "non-chemotherapy" treatment option for patients with FL, both in the frontline and relapsed/refractory settings. ${ }^{16,17}$ Emerging data from phase $1 / 2$ clinical trials also show promising results with the combination of lenalidomide plus obinutuzumab. ${ }^{18-20}$ Building on these data, the phase 1b/2 GO29834 clinical trial combined pola with obinutuzumab and lenalidomide (pola-G-len) in patients with relapsed/refractory FL (Table 1). Interim results were presented at the American Society of Hematology 2019 annual meeting (data cut-off August 2019). ${ }^{21}$ Patients in the dose-expansion cohort received induction treatment with six 28-day cycles of pola $1.4 \mathrm{mg} / \mathrm{kg}$ on day 1 , lenalidomide $20 \mathrm{mg}$ daily on days 1-21, and obinutuzumab $1000 \mathrm{mg}$ on days 1,8 , and 15 of cycle 1 and day 1 of subsequent cycles. In responding patients, a maintenance phase followed with lenalidomide $10 \mathrm{mg}$ daily on days $1-21$ of each 28 -day cycle for 12 months and obinutuzumab every 2 months for 24 months. For the 56 safety-evaluable patients, median age was 62 years (range, 32-87), 98\% had ECOG PS 0-1, $16 \%$ had bulky disease, 55\% had Follicular Lymphoma International Prognostic Index (FLIPI) $\geq 3$, median number of prior treatments was 3 (range, $1-7$ ) with $59 \%$ refractory to last treatment, and 25\% had POD24. The most common grade 3-4 AEs were neutropenia (50\%), thrombocytopenia (23\%), infections (16\%), and anemia (14\%). G-CSF was used in $55 \%$ of the patients during induction and $36 \%$ during maintenance. PN occurred in $30 \%$ of the patients. AEs leading to any dose reductions or drug discontinuations occurred in $34 \%$ and $30 \%$, respectively. For the 46 efficacy-evaluable patients, the EOT ORR was $76 \%$ with $63 \%$ attaining $\mathrm{CR}$. The ORR/CR rate with pola-G-len were particularly remarkable in high-risk patients: $70 \%$ / $70 \%$ in high-risk FLIPI $(n=26), 55 \% / 45 \%$ in patients with POD24 $(n=11), 68 \% / 60 \%$ in patients refractory to last treatment $(n=25)$, and $75 \% / 71 \%$ in patients with $\geq 3$ prior therapies $(n=24)$. With a median follow up of 15 months, the 12 -month PFS was $83 \%$. These results are certainly encouraging but longer follow-up and validation in other studies are required.

\section{Frontline Setting}

In the frontline setting, pola was evaluated in combination with cyclophosphamide, doxorubicin, and prednisone plus either rituximab or obinutuzumab (R-CHP or G-CHP) in a phase $1 \mathrm{~b} / 2$ clinical trial (Table 1$)^{22}$ The dose-escalation portion included any type of B-cell NHL whereas the dose-expansion portion was limited to patients with previously untreated DLBCL. Pola was administered on day 2 of the first two cycles, and on day 1 of subsequent cycles 
along with standard-dose CHP plus rituximab or obinutuzumab every 3 weeks for 6 or 8 cycles (per investigator discretion). The RP2D of pola was $1.8 \mathrm{mg} / \mathrm{kg}$. For the 66 patients with previously untreated DLBCL who received the RP2D (45 patients with R-CHP and 21 with G-CHP), median age was 68 years $(70 \%>65$ years $)$, ECOG PS was 2 in $29 \%$, and $65 \%$ had International Prognostic Index (IPI) of 3-5. The most common grade $\geq 3$ AEs at the RP2D were neutropenia (30\%), neutropenic fever (18\%), and thrombocytopenia (9\%). PN occurred in $41 \%$ of the patients treated at the RP2D and was grade 1 in $27 \%$, grade 2 in $11 \%$, and grade 3 in $3 \%$. As expected, PN grade 2 or 3 was more common in patients who received $>6$ cycles $(19 \%$ and $5 \%$, respectively) compared with those who received 6 cycles ( $6 \%$ and $3 \%$, respectively). PN resolved in the majority of patients $(74 \%)$ in a median of 2 months for grade 1 and a median of 5 months for grade $2-3$. Of the 7 patients with persistent $\mathrm{PN}$, one had grade 2 and the rest had grade 1 . Seven patients (11\%) discontinued treatment (received $<6$ cycles) due to an $\mathrm{AE}$ (4 patients with G-CHP and 3 with R-CHP). Doxorubicin and cyclophosphamide dose reductions were uncommon (5\% and $2 \%$, respectively). Two treatment-related deaths were reported (one complication of atrial fibrillation and one septic shock). The ORR for patients treated at the RP2D was $89 \%$ including $77 \%$ attaining CR, with PFS at 24 months of $83 \%$. No significant differences in response rates and 12-month PFS were seen between the R-CHP and G-CHP cohorts. PFS was similar regardless of $\mathrm{COO}$ by GEP (available for $\mathrm{n}=51$ : $16 \mathrm{ABC}, 28 \mathrm{GCB}, 7$ unclassifiable) and MYC/BCL2 double-expression status (available for 41 patients, present in 13 patients). Bearing in mind the small number of patients treated on this trial and the limitations of cross-trial comparisons, these results compared favorably with those of the GOYA trial, which randomized patients with previously untreated DLBCL to 6 or 8 cycles of cyclophosphamide, doxorubicin, vincristine, and prednisone plus either rituximab or obinutuzumab (R-CHOP or G-CHOP). ${ }^{23}$ Overall, patients treated on GOYA had a more favorable risk-profile as they were younger (median age of 62 vs 68 years), had better ECOG PS (2-3 in $12-14 \%$ vs 2 in 29\%), and had lower risk disease by IPI (3-5 in $43-47 \%$ vs $65 \%$ ). Pola plus $\mathrm{R}-\mathrm{CHP} / \mathrm{G}-\mathrm{CHP}$ resulted in higher CR rate and 1-year PFS compared with R-CHOP/G-CHOP: $77 \%$ vs 58\% and $91 \%$ vs $81 \%$, respectively. In terms of AEs, treatment with pola plus R-CHP/G-CHP resulted in higher rates of grade 3-5 thrombocytopenia ( $9 \%$ vs $1-4 \%$ ) and all grade PN (41\% vs $13 \%$ ) without an increased risk of grade 3-5 neutropenia $(30 \%$ vs $46-55 \%)$, neutropenic fever (18\% vs $15-18 \%$ ) or infections (15\% vs $16-19 \%)$. Although these results are encouraging, they require confirmation in a randomized clinical trial. This is the aim of the recently concluded POLARIX trial, which randomized 875 patients with previously untreated DLBCL to pola plus R-CHP vs R-CHOP (Table 2). The results of POLARIX are eagerly awaited.

None of the previously mentioned clinical trials were able to establish predictive or prognostic biomarkers for response to treatment with pola. CD79b expression by IHC was present in virtually all cases of DLBCL and FL, and even in the very few cases with negative expression by IHC ( $4 \%$ in one study), ${ }^{14}$ CD79b RNA expression was present. ${ }^{13,14,22} \mathrm{CD} 79 \mathrm{~b}$ expression levels in patients with DLBCL or FL did not correlate with response to treatment with pola alone or when combined with chemoimmunotherapy. ${ }^{13,14,22}$ Response to treatment with pola was similar across DLBCL subtypes regardless of $\mathrm{COO}^{13,14}$ or $\mathrm{MYC} / \mathrm{BCL} 2$ double-expression status, ${ }^{14,22}$ albeit the relatively small number of patients in each trial limits any firm conclusions.

\section{Clinical Practice Points}

The FDA-approved dose of pola is $1.8 \mathrm{mg} / \mathrm{kg}$ administered in combination with BR every 3 weeks for 6 cycles. Pola is administered intravenously over 90 minutes with premedication with an antipyretic and an antihistamine. Subsequent doses may be administered over 30 minutes if prior infusions were tolerated. ${ }^{24}$ Infusion reactions were reported in $7 \%$ of patients treated with pola-BR/pola-BG (despite premedications) with one-third of the cases being grade $2(25 \%)$ or $3(8 \%)$.

As discussed previously, myelosuppression is common with pola especially when combined with chemotherapy or other myelosuppressive agents. Grade 3-4 neutropenia occurred in $46 \%$ of patients with DLBCL treated with pola-BR. Prophylaxis with G-CSF was optional on the GO29365 clinical trial but $42 \%$ of patients treated with pola-BR received primary G-CSF prophylaxis. When pola is given with $\mathrm{BR}$, prophylaxis for Pneumocystis jiroveci pneumonia and herpesvirus is recommended.

Similar to other MMAE-containing ADCs (such as brentuximab), $\mathrm{PN}$ is common with pola and is dose- and duration-dependent. Importantly, $\mathrm{PN}$ is common in patients with DLBCL treated with vinca alkaloids and/or platinum agents, and patients with baseline $\mathrm{PN}$ of grade $\geq 2$ 
Table 2 Ongoing Clinical Trials with Polatuzumab in Patients with B-Cell NHL

\begin{tabular}{|c|c|c|c|c|c|}
\hline $\begin{array}{l}\text { NCT\# (Study } \\
\text { Name) }\end{array}$ & Phase & NHL Subtype(s) & Treatments & Site(s) & $\begin{array}{l}\text { Recruiting } \\
\text { (Yes/No) }\end{array}$ \\
\hline NCT0423।877 & 1 & $\begin{array}{l}\text { Previously untreated } \\
\text { aggressive B-cell + PMBCL }\end{array}$ & DA-EPCH-R + pola & US & No \\
\hline $\begin{array}{l}\text { NCT03274492 } \\
(\text { POLARIX) }\end{array}$ & 3 & Previously untreated DLBCL & Pola-R-CHP vs R-CHOP & US & $\begin{array}{l}\text { Fully } \\
\text { recruited }\end{array}$ \\
\hline \multirow[t]{2}{*}{$\begin{array}{l}\text { NCT0367I0I8 } \\
(\text { GO405I6) }\end{array}$} & $\mathrm{Ib} / 2$ & $\mathrm{R} / \mathrm{R} \mathrm{FL}$ & $\begin{array}{l}\text { Mosunetuzumab (M) + pola vs R-CHOP, R-CVP, } \\
\text { or BG }\end{array}$ & \multirow[t]{2}{*}{ US } & \multirow[t]{2}{*}{ Yes } \\
\hline & & R/R DLBCL & Pola-BR vs $M$ monotherapy vs $M+$ pola & & \\
\hline NCT04236I4I & 3 & R/R DLBCL & Pola-BR vs BR & China & No \\
\hline $\begin{array}{l}\text { NCT04I82204 } \\
\text { (POLARGO) }\end{array}$ & 3 & R/R DLBCL & Pola-R-GemOx vs R-GemOx & International & Yes \\
\hline NCT02600897 & $\mathrm{Ib} / 2$ & $\begin{array}{l}\text { R/R FL } \\
R / R \text { DLBCL }\end{array}$ & $\begin{array}{l}\text { Pola-lenalidomide }+ \text { obinutuzumab }(\mathrm{FL}) \text { or } \\
\text { rituximab }(\mathrm{DLBCL})\end{array}$ & International & Yes \\
\hline NCT026II 323 & $\mathrm{Ib} / 2$ & $\begin{array}{l}\text { R/R FL } \\
R / R \text { DLBCL }\end{array}$ & $\begin{array}{l}\text { Pola-venetoclax }+ \text { obinutuzumab }(\mathrm{FL}) \text { or } \\
\text { rituximab }(\mathrm{DLBCL})\end{array}$ & International & Yes \\
\hline NCT03533283 & $\mathrm{Ib}$ & R/R B-cell NHL & $\begin{array}{l}\text { RO7082859 (CD20xCD3 bispecific) + pola, or } \\
\text { RO7082859 + atezolizumab }\end{array}$ & Europe & Yes \\
\hline \multirow[t]{2}{*}{ NCT03677I4I } & $\mathrm{Ib}$ & $\mathrm{R} / \mathrm{R} \mathrm{NHL}$ & M-CHOP, M-CHP-pola & \multirow[t]{2}{*}{ International } & \multirow[t]{2}{*}{ Yes } \\
\hline & 2 & Previously untreated DLBCL & M-CHOP or M-CHP-pola vs pola-R-CHP & & \\
\hline NCT02729896 & $\mathrm{Ib} / 2$ & $\begin{array}{l}\text { R/R DLBCL } \\
R / R \text { FL }\end{array}$ & $\begin{array}{l}\text { Pola-atezolizumab + obinutuzumab }(\mathrm{FL}) \text { or } \\
\text { rituximab }(\mathrm{DLBCL})\end{array}$ & International & No \\
\hline
\end{tabular}

Abbreviations: BG, obinutuzumab and bendamustine; BR, bendamustine and rituximab; $\mathrm{CHP}$, cyclophosphamide, doxorubicin and prednisone; DA-EPCH-R, dose-adjusted rituximab, etoposide, cyclophosphamide, and doxorubicin; DLBCL, diffuse large B-cell lymphoma; FL, follicular lymphoma; M, mosunetuzumab (CD20xCD3 bispecific antibody); NHL, non-Hodgkin lymphoma; Pola, polatuzumab; PMBCL, primary mediastinal large B-cell lymphoma; R-CHOP, rituximab, cyclophosphamide, doxorubicin, vincristine and prednisone; R-CVP, rituximab, cyclophosphamide, vincristine and prednisone; R-GemOx, rituximab, gemcitabine, and oxaliplatin; R/R, relapsed/refractory.

were excluded from the previously described trials of pola. When dosed at $1.8 \mathrm{mg} / \mathrm{kg}$, pola-induced PN occurred in $40-45 \%$ of the patients with the majority reported being grade $1(27-28 \%)$ whereas grade 2 and 3 occurred in $11-15 \%$ and $0-3 \%$, respectively. PN resolved in $59-75 \%$ of the patients with a median time to resolution of 2 months for grade 1 and 5 months for grades $2-3$. For patients with pola-induced grade $\geq 2 \mathrm{PN}$, holding treatment with pola is recommended until PN improves to grade $\leq 1$. Pola may be resumed with the next cycle at a permanently reduced dose of $1.4 \mathrm{mg} / \mathrm{kg}$ if the $\mathrm{PN}$ resolves to grade $\leq 1$ by 2 weeks. Treatment with pola should be discontinued if the PN does not resolve to grade $\leq 1$ by 2 weeks, PN grade $\geq 2$ recurs despite dose reduction, or the PN is grade $4 .{ }^{24}$

Grade 3 and 4 transaminase elevations were each reported in $1.9 \%$ of the patients treated with pola on the GO29365 clinical trial. Based on the US
Prescribing Information, pola use should be avoided in patients with moderate to severe hepatic impairment (total bilirubin $>1.5$ upper limit of normal [ULN]) because hepatic impairment could increase the exposure to MMAE. No dose adjustment is required in patients with mild hepatic impairment (total bilirubin $>1-1.5$ times ULN). ${ }^{24}$ The effect of severe renal impairment $(\mathrm{CrCl}<30 \mathrm{~mL} /$ minute) or end-stage renal disease on the pharmacokinetics of pola is unknown, whereas no clinically significant pharmacokinetic differences were observed based in mild to moderate renal impairment ( $\mathrm{CrCl} 30-89 \mathrm{~mL} /$ minute).

Anti-pola antibodies were detected in $2.6 \%$ of polatreated patients, ${ }^{24}$ including antibodies against both the antibody and the linker-MMAE components of pola. ${ }^{12}$ The significance of these anti-pola antibodies on pola's safety or efficacy is unclear at this point. ${ }^{24}$ 


\section{Current Role and Future Directions In DLBCL}

In heavily pretreated and transplantation-ineligible patients with DLBCL treated on the GO29365 trial, pola-BR resulted in an EOT ORR of $45 \%$ including CR in $40 \%$, best ORR of $70 \%$ including CR in 58\%, and median PFS, DOR, and OS of 10,13, and 12 months, respectively. These results have established pola-BR as a new and effective option for a patient population with otherwise very limited treatment options. The incorporation of pola into the treatment paradigm of patients with relapsed/refractory DLBCL is certainly welcomed. However, there are important points about the GO29365 trial that need to be highlighted. First, the treatment the comparator arm received (BR) had very modest clinical activity (ORR and CR rate of only 18\%). Furthermore, this relatively small trial was underpowered to detect the survival benefit seen with pola-BR. POLARGO (NCT04182204) is an ongoing Phase 3 clinical trial randomizing patients with relapsed/refractory DLBCL to rituximab, gemcitabine, and oxaliplatin alone or in combination with pola (Table 2). POLARGO will be an important trial to confirm the clinical activity of pola when combined with more effective chemotherapy and its role in transplantationeligible patients. Second, the role of pola-BR in patients whose DLBCL progressed/relapsed after autologous SCT and/or chimeric antigen receptor T-cell therapy (CAR-T) is currently undefined as only $25 \%$ of patients treated with pola-BR on GO29365 had undergone prior autologous SCT and none had received prior CAR-T. Similarly, the role of pola-BR as a bridging therapy to either autologous SCT or CAR-T is unknown, particularly the effect of prolonged treatment with bendamustine on stem cell mobilization or quality of the CAR-T product in heavily pretreated patients. Finally, it is unclear whether pola-BR is equally effective in patients with transformed FL or double- and triple-hit lymphoma as these patients were not included on GO29365.

Table 2 lists other planned or ongoing clinical trials of pola in DLBCL. As previously mentioned, the recently concluded phase 3 POLARIX trial will define if there is a role for pola in combination with chemotherapy (R-CHP) in patients with previously untreated DLBCL. NCT04231877 is a phase 1 clinical trial combining pola with dose-adjusted rituximab, etoposide, cyclophosphamide, doxorubicin, and prednisone (DA-EPCH-R) in patients with aggressive B-cell lymphoma and primary mediastinal large B-cell lymphoma (PMBL). Of note, preclinical studies show activity for pola in cell lines and xenograft models of Burkitt and PMBL. ${ }^{25}$
NCT03677141 is an ongoing phase $1 \mathrm{~b} / 2$ clinical trial that will include a randomized comparison of mosunetuzumab (a CD20/CD3 bispecific antibody) in combination with CHOP (M-CHOP), M-CHP-pola, and R-CHP-pola in patients with previously untreated DLBCL. GO40516 (NCT03671018) is a phase $1 b / 2$ clinical trial that investigates the combination of mosunetuzumab plus pola vs mosunetuzumab monotherapy vs pola-BR in patients with relapsed/refractory DLBCL.

\section{In FL}

There is currently no defined role for pola in FL. Pola monotherapy or when combined with rituximab resulted in short-lived remissions, and its addition to BR did not improve response rates or PFS compared with BR alone, albeit with very short follow-up. Pola has the potential to be combined with several targeted agents including lenalidomide as discussed previously. Mosunetuzumab plus pola is also being evaluated in patients with relapsed/ refractory FL (GO40516, NCT03671018).

Pola is also being studied in combination with atezolizumab and RO7082859 (a CD20/CD3 bispecific antibody) in relapsed/refractory FL or DLBCL (NCT02729896, NCT03533283). Based on preclinical data showing increased BCL-XL expression as a potential resistance mechanism to pola, and that combining pola with a BCL2 inhibitor (navitoclax) enhances its in vivo activity, ${ }^{1}$ pola is being studied in combination with venetoclax plus rituximab or obinutuzumab in relapsed/refractory FL or DLBCL (NCT02611323).

\section{Other Anti-CD79b ADCs}

Iladatuzumab vedotin (DCDS0780A) is another humanized anti-CD79b antibody conjugated to MMAE that has shown clinical activity in a small phase $1 \mathrm{~b}$ clinical trial in patients with NHL (60\% ORR including 43\% CR in 30 patients with relapsed/refractory DLBCL) ${ }^{26}$

\section{Conclusion}

The anti-CD79b ADC pola has shown promising activity in relapsed/refractory DLBCL and FL, and is currently FDA-approved in combination with bendamustine and rituximab in patients with relapsed/refractory DLBCL after at least two prior therapies. Myelosuppression and peripheral neuropathy are the main toxicities of pola. Pola has also shown encouraging preliminary results when combined with R-CHP in patients with previously untreated DLBCL and the results of the phase 3 trial of pola plus R-CHP vs R-CHOP will define if it has a role in 
the frontline treatment of DLBCL. Pola's role in FL is less clear at this point as the combinations studied so far have failed to show a benefit over approved therapies. Given the wide expression of its target (CD79b), unique mechanism of action, and good safety profile, pola is a suitable candidate for combination studies in B-cell NHL. The results of ongoing clinical trials to confirm pola's efficacy and investigate other potential combinations are eagerly awaited.

\section{Disclosure}

The authors report no conflicts of interest in this work.

\section{References}

1. Dornan D, Bennett F, Chen Y, et al. Therapeutic potential of an anti-CD79b antibody-drug conjugate, anti-CD79b-vc-MMAE, for the treatment of non-Hodgkin lymphoma. Blood. 2009;114 (13):2721-2729. doi:10.1182/blood-2009-02-205500

2. Dal Porto JM, Gauld SB, Merrell KT, Mills D, Pugh-Bernard AE, Cambier J. B cell antigen receptor signaling 101. Mol Immunol. 2004;41(6-7):599-613. doi:10.1016/j.molimm.2004.04.008

3. Chu PG, Arber DA. CD79: a review. Appl Immunohistochem Mol Morphol. 2001;9(2):97-106. doi:10.1097/00129039-200106000-00 001

4. Polson AG, Yu SF, Elkins K, et al. Antibody-drug conjugates targeted to CD79 for the treatment of non-Hodgkin lymphoma. Blood. 2007;110(2):616-623. doi:10.1182/blood-2007-01-066704

5. Busman-Sahay K, Drake L, Sitaram A, Marks M, Drake JR. Cis and trans regulatory mechanisms control AP2-mediated B cell receptor endocytosis via select tyrosine-based motifs. PLoS One. 2013;8(1): e54938. doi:10.1371/journal.pone.0054938

6. Cabezudo E, Carrara P, Morilla R, Matutes E. Quantitative analysis of CD79b, CD5 and CD19 in mature B-cell lymphoproliferative disorders. Haematologica. 1999;84(5):413-418.

7. D’Arena G, Musto P, Cascavilla N, Dell'Olio M, Di Renzo N, Carotenuto M. Quantitative flow cytometry for the differential diagnosis of leukemic B-cell chronic lymphoproliferative disorders. Am $J$ Hematol. 2000;64(4):275-281. doi:10.1002/1096-8652(200008) 64:4<275::aid-ajh7>3.0.co;2-y

8. Pfeifer M, Zheng B, Erdmann T, et al. Anti-CD22 and anti-CD79B antibody drug conjugates are active in different molecular diffuse large B-cell lymphoma subtypes. Leukemia. 2015;29(7):1578-1586. doi:10.1038/leu.2015.48

9. Fuh FK, Looney C, Li D, et al. Anti-CD22 and anti-CD79b antibody-drug conjugates preferentially target proliferating B cells. Br J Pharmacol. 2017;174(8):628-640. doi:10.1111/bph.13697

10. Schmitz R, Wright GW, Huang DW, et al. Genetics and pathogenesis of diffuse large B-cell lymphoma. N Engl J Med. 2018;378 (15):1396-1407. doi:10.1056/NEJMoa1801445

11. Allan JN, Bhavsar EB, Vaisitti T, et al. CD79b expression in Richter's transformation. Blood. 2019;134(Supplement_1):4279. doi:10.1182/blood-2019-128305

12. Palanca-Wessels MCA, Czuczman M, Salles G, et al. Safety and activity of the anti-CD79B antibody-drug conjugate polatuzumab vedotin in relapsed or refractory B-cell non-Hodgkin lymphoma and chronic lymphocytic leukaemia: a phase 1 study. Lancet Oncol. 2015;16(6):704-715. doi:10.1016/S1470-2045(15)70128-2
13. Morschhauser F, Flinn IW, Advani R, et al. Polatuzumab vedotin or pinatuzumab vedotin plus rituximab in patients with relapsed or refractory non-Hodgkin lymphoma: final results from a phase 2 randomised study (ROMULUS). Lancet Haematol. 2019;6(5):e254e265. doi:10.1016/S2352-3026(19)30026-2

14. Sehn LH, Herrera AF, Flowers CR, et al. Polatuzumab vedotin in relapsed or refractory diffuse large B-cell lymphoma. J Clin Oncol. 2019;38(2):JCO1900172. doi:10.1200/JCO.19.00172

15. Sehn LH, Kamdar M, Herrera AF, et al. Randomized phase 2 trial of polatuzumab vedotin (pola) with bendamustine and rituximab (BR) in relapsed/refractory (r/r) FL and DLBCL. J Clin Oncol. 2018;36 (15_suppl):7507. doi:10.1200/JCO.2018.36.15_suppl.7507

16. Leonard JP, Trneny M, Izutsu K, et al. AUGMENT: a Phase III study of lenalidomide plus rituximab versus placebo plus rituximab in relapsed or refractory indolent lymphoma. J Clin Oncol. 2019;37 (14):1188-1199. doi:10.1200/JCO.19.00010

17. Morschhauser F, Fowler NH, Feugier P, et al. Rituximab plus lenalidomide in advanced untreated follicular lymphoma. $N$ Engl J Med. 2018;379(10):934-947. doi:10.1056/NEJMoa1805104

18. Morschhauser F, Le Gouill S, Feugier P, et al. Obinutuzumab combined with lenalidomide for relapsed or refractory follicular B-cell lymphoma (GALEN): a multicentre, single-arm, phase 2 study. Lancet Haematol. 2019;6(8):e429-e437. doi:10.1016/S2352-3026(19)30089-4

19. Fowler NH, Nastoupil LJ, Chin C, et al. A Phase I/II study of lenalidomide plus obinutuzumab in relapsed indolent lymphoma. Blood. 2019;134(Supplement_1):348. doi:10.1182/blood-2019-131 045

20. Nastoupil LJ, Westin JR, Hagemeister FB, et al. Results of a Phase II study of obinutuzumab in combination with lenalidomide in previously untreated, high tumor burden follicular lymphoma (FL). Blood. 2019;134(Supplement_1):125. doi:10.1182/blood-2019-129 422

21. Diefenbach C, Kahl BS, Banerjee L, et al. Polatuzumab vedotin plus obinutuzumab and lenalidomide in patients with relapsed/refractory follicular lymphoma: primary analysis of the full efficacy population in a Phase Ib/II Trial. Blood. 2019;134(Supplement_1):126. doi:10.1182/blood-2019-123669

22. Tilly H, Morschhauser F, Bartlett NL, et al. Polatuzumab vedotin in combination with immunochemotherapy in patients with previously untreated diffuse large B-cell lymphoma: an open-label, nonrandomised, phase 1b-2 study. Lancet Oncol. 2019;20(7):998-1010. doi:10.1016/S1470-2045(19)30091-9

23. Vitolo U, Trneny M, Belada D, et al. Obinutuzumab or rituximab plus cyclophosphamide, doxorubicin, vincristine, and prednisone in previously untreated diffuse large b-cell lymphoma. J Clin Oncol. 2017;35(31):3529-3537. doi:10.1200/JCO.2017.73.3402

24. Genentech Inc. Polivy ${ }^{\mathrm{TM}}$ (Polatuzumab vedotin-piiq) for injection, for intravenous use: US Prescribing Information; 2019. Available from: https://www.accessdata.fda.gov/drugsatfda_docs/label/2019/ 761121s000lbl.pdf. Accessed February 21, 2020.

25. Audino AN, Barth MJ, Tiwari A, et al. Polatuzumab vedotin, an antibody-drug conjugate targeting CD79b, is a highly active agent against burkitt lymphoma and primary mediastinal B-cell lymphoma. Blood. 2019;134(Supplement_1):3963. doi:10.1182/blood-2019-128580

26. Herrera A, Patel M, Burke J, et al. A Phase I study of the anti-CD79b THIOMAB antibody-drug conjugate DCDS0780A in patients with relapsed or refractory B-cell non-Hodgkin's lymphoma. Blood. 2017;130:4140. 


\section{Publish your work in this journal}

OncoTargets and Therapy is an international, peer-reviewed, open access journal focusing on the pathological basis of all cancers, potential targets for therapy and treatment protocols employed to improve the management of cancer patients. The journal also focuses on the impact of management programs and new therapeutic agents and protocols on patient perspectives such as quality of life, adherence and satisfaction. The manuscript management system is completely online and includes a very quick and fair peer-review system, which is all easy to use. Visit http://www.dovepress.com/ testimonials.php to read real quotes from published authors.

Submit your manuscript here: https://www.dovepress.com/oncotargets-and-therapy-journal 